\title{
Implicit Active Contour Model with Local and Global Intensity Fitting Energies
}

\author{
Xiaozeng $\mathrm{Xu}^{1,2}$ and Chuanjiang $\mathrm{He}^{1}$ \\ ${ }^{1}$ College of Mathematics and Statistics, Chongqing University, Chongqing 401331, China \\ ${ }^{2}$ School of Mathematics and Statistics, Chongqing University of Technology, Chongqing 400054, China \\ Correspondence should be addressed to Xiaozeng Xu; xuxz@cqut.edu.cn
}

Received 19 December 2012; Accepted 28 March 2013

Academic Editor: Oluwole Daniel Makinde

Copyright (C) $2013 \mathrm{X}$. Xu and C. He. This is an open access article distributed under the Creative Commons Attribution License, which permits unrestricted use, distribution, and reproduction in any medium, provided the original work is properly cited.

\begin{abstract}
We propose a new active contour model which integrates a local intensity fitting (LIF) energy with an auxiliary global intensity fitting (GIF) energy. The LIF energy is responsible for attracting the contour toward object boundaries and is dominant near object boundaries, while the GIF energy incorporates global image information to improve the robustness to initialization of the contours. The proposed model not only can provide desirable segmentation results in the presence of intensity inhomogeneity but also allows for more flexible initialization of the contour compared to the RSF and LIF models, and we give a theoretical proof to compute a unique steady state regardless of the initialization; that is, the convergence of the zero-level line is irrespective of the initial function. This means that we can obtain the same zero-level line in the steady state, if we choose the initial function as a bounded function. In particular, our proposed model has the capability of detecting multiple objects or objects with interior holes or blurred edges.
\end{abstract}

\section{Introduction}

Implicit active contour models have been extensively studied and successfully used in image segmentation [1-3]. The basic idea is to evolve a contour under some constraints to extract the desired object. According to the nature of constraints, the existing active contour models can be categorised into two classes: edge-based models [4-7], and region-based models [8-17]. Each of them has its own pros and cons; the choice of them in applications depends on different characteristics of images. In this study, we focus on region-based models and consider images with intensity inhomogeneity.

Unlike edge-based models that utilize typically an edge indicator depending on image gradient to perform contour extraction, region-based models usually use global and/or local statistics inside regions rather than gradient on edges to find a partition of image domain. They generally have better performances in the presence of weak or discontinuous boundaries than edge-based models. Early popular regionbased models tend to rely on intensity homogeneous (roughly constant or smooth) statistics in each of the regions to be segmented. Therefore, they either lack the ability to deal with intensity inhomogeneity like the PC (piecewise constant) model [8] or are excessively expensive in computation like the PS (piecewise smooth) model [9].

To handle intensity inhomogeneity efficiently, some localized region-based models [11-16] have been proposed recently. For example, Li et al. [12] recently proposed a regionscalable fitting (RSF) active contour model (originally termed as local binary fitting (LBF) model [11]). The RSF model draws upon intensity information in spatially varying local regions depending on a scale parameter, so it is able to deal with intensity inhomogeneity efficiently. Very recently, Zhang et al. [15] proposed a novel active contour model driven by local image fitting energy, which also can handle intensity inhomogeneity efficiently. The experimental results in [15] show that this model is more efficient than the RSF model, while yielding similar results. However, these two models easily get stuck in local minimums for the most of contour initializations. This makes it need user intervention to define the initial contours professionally.

In this study, based on the PC model [8] and RSF model [12], we propose a new active contour model, which integrates a local intensity fitting (LIF) energy with an auxiliary global 
intensity fitting (GIF) energy. The LIF energy is responsible for attracting the contour toward object boundaries and is dominant near object boundaries, while the GIF energy incorporates global image information to improve the robustness to initialization of the contours. The proposed model can efficiently handle intensity inhomogeneity, while allowing for more flexible initialization and maintaining the subpixel accuracy.

The remainder of this paper is organized as follows. Section 2 briefly reviews the PC model [8] and RSF model [12]. Section 3 introduces the proposed model. Section 4 presents experimental results using a set of synthetic and real images. This paper is summarized in Section 5.

\section{Related Works}

2.1. Piecewise Constant Model by Chan and Vese. In [8], Chan and Vese (CV) proposed a region-based active contour model that relies on intensity homogeneous (roughly constant) statistics in each of the regions to be segmented. In the CV model, a contour $C$ is represented implicitly by the zero-level set of a Lipschitz function $\phi: \Omega \rightarrow \mathbf{R}$, which is called a level set function. In what follows, we let the level set function $\phi$ take positive and negative values inside and outside the contour $C$, respectively.

Let $I: \Omega \subset \mathbf{R}^{2} \rightarrow \mathbf{R}$ be an input image, and let $H_{\varepsilon}$ be the regularized Heaviside function; the energy functional of the $\mathrm{CV}$ model is defined as

$$
\begin{aligned}
E^{\mathrm{CV}}\left(c_{1}, c_{2}, \phi\right)= & \lambda_{1} \int_{\Omega}\left|I-c_{1}\right|^{2} H_{\varepsilon}(\phi) d \mathbf{x} \\
& +\lambda_{1} \int_{\Omega}\left|I-c_{2}\right|^{2}\left(1-H_{\varepsilon}(\phi)\right) d \mathbf{x} \\
& +\nu \int_{\Omega}\left|\nabla H_{\varepsilon}(\phi)\right| d \mathbf{x},
\end{aligned}
$$

where $\lambda_{1}, \lambda_{2}>0, v>0$ are constants. The regularized version of $H(z)$ is chosen as

$$
H_{\varepsilon}(z)=\frac{1}{2}\left(1+\frac{2}{\pi} \arctan \left(\frac{z}{\varepsilon}\right)\right) .
$$

$c_{1}$ and $c_{2}$ are the global averages of the image intensities in the region $\{\mathbf{x}: \phi(\mathbf{x})>0\}$ and $\{\mathbf{x}: \phi(\mathbf{x})<0\}$, respectively; that is,

$$
\begin{gathered}
c_{1}(\phi)=\frac{\int_{\Omega} I(\mathbf{x}) H_{\varepsilon}(\phi(\mathbf{x})) d \mathbf{x}}{\int_{\Omega} H_{\varepsilon}(\phi(\mathbf{x})) d \mathbf{x}}, \\
c_{2}(\phi)=\frac{\int_{\Omega} I(\mathbf{x})\left(1-H_{\varepsilon}(\phi(\mathbf{x}))\right) d \mathbf{x}}{\int_{\Omega}\left(1-H_{\varepsilon}(\phi(\mathbf{x}))\right) d \mathbf{x}} .
\end{gathered}
$$

The solution of the CV model in fact leads to a piecewise constant segmentation of the original image $I(\mathbf{x})$ :

$$
I^{G}(\mathbf{x})=c_{1} H_{\varepsilon}(\phi(\mathbf{x}))+c_{2}\left(1-H_{\varepsilon}(\phi(\mathbf{x}))\right),
$$

where $c_{1}$ and $c_{2}$ are the averages of the image intensities in the region $\{\mathbf{x}: \phi(\mathbf{x})>0\}$ and $\{\mathbf{x}: \phi(\mathbf{x})<0\}$, respectively. Such constants may be far away from the original image data, if the intensities outside or inside the contour $C=\{\mathbf{x}: \phi(\mathbf{x})=0\}$ are not homogeneous. As a result, the CV model generally fails to segment images with intensity inhomogeneity.
2.2. Region-Scalable Fitting Model. In order to improve the performance of the global CV model [8] and the PS model [9] on images with inhomogeneity, Li et al. $[11,12]$ recently proposed a novel region-based active contour model. They introduced a kernel function and defined the following energy functional:

$$
\begin{aligned}
E^{\mathrm{RSF}}\left(f_{1}, f_{2}, \phi\right)=\lambda_{1} \int\left[\int\right. & K_{\sigma}(\mathbf{x}-\mathbf{y})\left|I(\mathbf{y})-f_{1}(\mathbf{x})\right|^{2} \\
& \left.\times H_{\varepsilon}(\phi(\mathbf{y})) d \mathbf{y}\right] d \mathbf{x} \\
& +\lambda_{2} \int\left[\int K_{\sigma}(\mathbf{x}-\mathbf{y})\left|I(\mathbf{y})-f_{1}(\mathbf{x})\right|^{2}\right. \\
& \left.\times\left(1-H_{\varepsilon}(\phi(\mathbf{y}))\right) d \mathbf{y}\right] d \mathbf{x} \\
& +\nu \int_{\Omega}\left|\nabla H_{\varepsilon}(\phi(\mathbf{x}))\right| d \mathbf{x} \\
& +\mu \int_{\Omega} \frac{1}{2}(|\nabla \phi(\mathbf{x})|-1)^{2} d \mathbf{x},
\end{aligned}
$$

where $K_{\sigma}$ is a Gaussian kernel with standard deviation $\sigma$, and $f_{1}(\mathbf{x})$ and $f_{2}(\mathbf{x})$ are two smooth functions that approximate the local image intensities inside and outside the contour, respectively. They are computed by

$$
\begin{gathered}
f_{1}(\mathbf{x})=\frac{\int_{\Omega} K_{\sigma}(\mathbf{x}-\mathbf{y}) I(\mathbf{y}) H_{\varepsilon}(\phi(\mathbf{y})) d \mathbf{y}}{\int_{\Omega} K_{\sigma}(\mathbf{x}-\mathbf{y}) H_{\varepsilon}(\phi(\mathbf{y})) d \mathbf{y}}, \\
f_{2}(\mathbf{x})=\frac{\int_{\Omega} K_{\sigma}(\mathbf{x}-\mathbf{y}) I(\mathbf{y})\left(1-H_{\varepsilon}(\phi(\mathbf{y}))\right) d \mathbf{y}}{\int_{\Omega} K_{\sigma}(\mathbf{x}-\mathbf{y})\left(1-H_{\varepsilon}(\phi(\mathbf{y}))\right) d \mathbf{y}} .
\end{gathered}
$$

The solution of the RSF model leads to a piecewise smooth approximation of the original image $I(\mathbf{x})$ :

$$
I^{L}(\mathbf{x})=f_{1}(\mathbf{x}) H_{\varepsilon}(\phi(\mathbf{x}))+f_{2}(\mathbf{x})\left(1-H_{\varepsilon}(\phi(\mathbf{x}))\right),
$$

where the smooth functions $f_{1}(\mathbf{x})$ and $f_{2}(\mathbf{x})$ are computed by (6). $f_{1}(\mathbf{x})$ and $f_{2}(\mathbf{x})$ are the averages of local intensities on the two sides of the contour, which are different from the constants $c_{1}$ and $c_{2}$ in the CV model, the averages of the image intensities on the two sides of the contour. Therefore, the RSF model can deal with intensity inhomogeneity efficiently. However, it is sensitive to contour initialization (initial locations, sizes, and shapes).

\section{The Proposed Model}

3.1. Description of Our Model. Given a positive constant $\omega$ $(0 \leq \omega \leq 1)$, from (4) and (7), we define the following energy functional:

$$
\begin{aligned}
E\left(\phi, c_{1}, c_{2}, f_{1}, f_{2}\right)= & \omega E^{G}(\phi)+(1-\omega) E^{L}(\phi) \\
= & \omega\left(\frac{1}{2} \int_{\Omega}\left|I(\mathbf{x})-I^{G}(\mathbf{x})\right|^{2} d \mathbf{x}\right) \\
& +(1-\omega)\left(\frac{1}{2} \int_{\Omega}\left|I(\mathbf{x})-I^{L}(x)\right|^{2} d \mathbf{x}\right),
\end{aligned}
$$


where

$$
\begin{gathered}
I^{G}(\mathbf{x})=c_{1} H_{\varepsilon}(\phi(\mathbf{x}))+c_{2}\left(1-H_{\varepsilon}(\phi(\mathbf{x}))\right), \\
I^{L}(\mathbf{x})=f_{1}(\mathbf{x}) H_{\varepsilon}(\phi(\mathbf{x}))+f_{2}(\mathbf{x})\left(1-H_{\varepsilon}(\phi(\mathbf{x}))\right) .
\end{gathered}
$$

Keeping $\phi$ fixed and minimizing the functional $E\left(\phi, c_{1}, c_{2}\right.$, $f_{1}, f_{2}$ ) with respect to $c_{1}, c_{2}, f_{1}$, and $f_{2}$, we have

$$
\begin{gathered}
c_{1}=\frac{\int_{\Omega} I(\mathbf{x}) H_{\varepsilon}(\phi(\mathbf{x})) d \mathbf{x}}{\int_{\Omega} H_{\varepsilon}(\phi(\mathbf{x})) d \mathbf{x}}, \\
c_{2}=\frac{\int_{\Omega} I(\mathbf{x})\left(1-H_{\varepsilon}(\phi(\mathbf{x}))\right) d \mathbf{x}}{\int_{\Omega}\left(1-H_{\varepsilon}(\phi(\mathbf{x}))\right) d \mathbf{x}}, \\
f_{1}(\mathbf{x})=\frac{\int_{\Omega} K_{\sigma}(\mathbf{x}-y) I(y) H_{\varepsilon}(\phi(y)) d y}{\int_{\Omega} K_{\sigma}(\mathbf{x}-y) H_{\varepsilon}(\phi(y)) d y}, \\
f_{2}(\mathbf{x})=\frac{\int_{\Omega} K_{\sigma}(\mathbf{x}-y) I(y)\left(1-H_{\varepsilon}(\phi(y))\right) d y}{\int_{\Omega} K_{\sigma}(\mathbf{x}-y)\left(1-H_{\varepsilon}(\phi(y))\right) d y} .
\end{gathered}
$$

Keeping $c_{1}, c_{2}, f_{1}$, and $f_{2}$ fixed and minimizing the functional $E\left(\phi, c_{1}, c_{2}, f_{1}, f_{2}\right)$ with respect to $\phi$, we obtain the corresponding gradient descent flow equation:

$$
\begin{aligned}
\frac{\partial \phi}{\partial t}=\delta_{\varepsilon}(\phi)\left[\omega\left(\left(I-I^{G}\right)\left(c_{1}-c_{2}\right)\right)\right. \\
\left.+(1-\omega)\left(\left(I-I^{L}\right)\left(f_{1}-f_{2}\right)\right)\right] \\
=\delta_{\varepsilon}(\phi)\left[\omega F^{G}(\phi)+(1-\omega) F^{L}(\phi)\right]=F(\phi),
\end{aligned}
$$

where

$$
\delta_{\varepsilon}(z)=H_{\varepsilon}^{\prime}(z)=\frac{1}{\pi} \frac{\varepsilon}{\varepsilon^{2}+z^{2}} .
$$

Like the CV and RSF models, our model is also implemented using an alternative procedure: for each iteration and the corresponding level set function $\phi^{n}$, we first compute the fitting values $c_{i}\left(\phi^{n}\right)$ and $f_{i}\left(\phi^{n}\right)$ and then obtain $\phi^{n+1}$ by minimizing $E\left(\phi, c_{1}\left(\phi^{n}\right), c_{2}\left(\phi^{n}\right), f_{1}\left(\phi^{n}\right), f_{2}\left(\phi^{n}\right)\right)$ with respect to $\phi$. This process is repeated until the zero-level set of $\phi^{n+1}$ is exactly on the object boundary.

In the following, we first discuss the properties of $F^{G}(\phi)$ and $F^{L}(\phi)$ and then analyze the behavior of (11).

3.2. Properties of $F^{G}(\phi)$ and $F^{L}(\phi)$. For the sake of simplicity, we state and prove the properties of $F^{G}(\phi)$ and $F^{L}(\phi)$ only for an image consisting of only two distinct gray levels:

$$
I(\mathbf{x})= \begin{cases}g_{1}, & \mathbf{x} \in \omega, \\ g_{2}, & \mathbf{x} \in \Omega \backslash \omega,\end{cases}
$$

where $g_{1}, g_{2} \geq 0$ with $g_{1} \neq g_{2}, \omega$ and $\Omega \backslash \omega$ represent the objects of interest and the background, respectively.
Theorem 1. Let $I(\mathbf{x})$ be an image by (13). Then one has

$$
F^{G}(\phi)=\left\{\begin{array}{c}
(M n-N m)\left(g_{1}-g_{2}\right)^{2} \\
\times\left[(M-N)(N-n) H_{\varepsilon}\right. \\
\left.\quad+N((M+n)-(m+N))\left(1-H_{\varepsilon}\right)\right] \\
\times\left(N^{2}(M-N)^{2}\right)^{-1}, \\
\text { in } \omega \\
-(M n-N m)\left(g_{1}-g_{2}\right)^{2} \\
\times\left[n(M-N) H_{\varepsilon}\right. \\
\left.+N(m-n)\left(1-H_{\varepsilon}\right)\right] \\
\times\left(N^{2}(M-N)^{2}\right)^{-1}, \\
\text { in } \Omega \backslash \omega,
\end{array}\right.
$$

and so,

$$
\operatorname{sign}\left(F^{G}(\phi)\right)= \begin{cases}+\operatorname{sign}(M n-N m), & \text { in } \omega, \\ -\operatorname{sign}(M n-N m), & \text { in } \Omega \backslash \omega,\end{cases}
$$

where

$$
\begin{gathered}
M=|\Omega|, \quad m=|\omega|, \quad N=|\{\phi>0\}|, \\
n=|\omega \cap\{\phi>0\}|,
\end{gathered}
$$

in which $|\Omega|$ is the area of the region $\Omega$ and similarly for others.

Remarks. (i) Due to the discrete nature of image, $|\Omega|$ is in fact the number of pixels in the image $I(\mathbf{x})$, and similarly to others.

(ii) The cases of $n=m, n=N$, and $n=0$ correspond to the zero-level lines of $\phi(\mathbf{x})$ which are encircling the object $(\omega)$, inside the object and within the background $(\Omega \backslash$ $\omega)$, respectively. The cases of $N \neq n \neq m$ and $N=n=$ $m$ correspond to the zero-level line of $\phi(\mathbf{x})$ that are partially inside the object and exactly on the object edge, respectively.

(iii) The significance of (15) is that the function $F^{G}(\phi)$ has the opposite sign in $\omega$ (object) and $\Omega \backslash \omega$ (background), respectively.

The proof of Theorem 1 is provided in Appendix A. The following result will be used in the proof of Theorem 7 , which guarantees that the evolution by (10) converges to a unique stable value after finite time.

Corollary 2. Let $I(\mathbf{x})$ be an image by (13). Then one has the following.

(i) If $M n-N m \geq 0$, then

$$
\begin{gathered}
F^{G}(\phi) \geq(M n-N m) \frac{\left(g_{1}-g_{2}\right)^{2}}{M^{4} / 4}, \quad \text { in } \omega, \\
F^{G}(\phi) \leq-(M n-N m) \frac{\left(g_{1}-g_{2}\right)^{2}}{M^{4} / 4}, \quad \text { in } \Omega \backslash \omega .
\end{gathered}
$$


(ii) If $M n-N m \leq 0$, then

$$
\begin{gathered}
F^{G}(\phi) \leq(M n-N m) \frac{\left(g_{1}-g_{2}\right)^{2}}{M^{4} / 4}, \quad \text { in } \omega, \\
F^{G}(\phi) \geq-(M n-N m) \frac{\left(g_{1}-g_{2}\right)^{2}}{M^{4} / 4}, \quad \text { in } \Omega \backslash \omega .
\end{gathered}
$$

The proof of Corollary 2 is given in Appendix A.

We call the property in Theorem 1 an adaptive signchanging property of $F^{G}(\phi)$. Such property also holds for $F^{L}(\phi)$, which can be expressed by the following theorem.

Theorem 3. Let $I(\mathbf{x})$ be an image given by (13). Then one has

$$
F^{L}(\phi)=\left\{\begin{array}{c}
(P q-Q p)\left(g_{1}-g_{2}\right)^{2} \\
\times\left[(P-Q)(Q-q) H_{\varepsilon}(\phi)\right. \\
\left.+Q((P+q)-(p+Q))\left(1-H_{\varepsilon}(\phi)\right)\right] \\
\times\left(Q^{2}(P-Q)^{2}\right)^{-1} \\
\text { in } \omega \\
-(P q-Q p)\left(g_{1}-g_{2}\right)^{2} \\
\times\left[q(P-Q) H_{\varepsilon}(\phi)\right. \\
\left.+Q(p-q)\left(1-H_{\varepsilon}(\phi)\right)\right] \\
\times\left(Q^{2}(P-Q)^{2}\right)^{-1} \\
\text { in } \Omega \backslash \omega
\end{array}\right.
$$

and so,

$$
\operatorname{sign}\left(F^{L}(\phi)\right)= \begin{cases}+\operatorname{sign}(P q-Q p), & \text { in } \omega, \\ -\operatorname{sign}(P q-Q p), & \text { in } \Omega \backslash \omega,\end{cases}
$$

where

$$
\begin{gathered}
P=\int_{\Omega} K_{\sigma}(\mathbf{x}-\xi) d \xi, \quad p=\int_{\omega} K_{\sigma}(\mathbf{x}-\xi) d \xi, \\
Q=\int_{\{\phi>0\}} K_{\sigma}(\mathbf{x}-\xi) d \xi, \\
q=\int_{\omega \cap\{\phi>0\}} K_{\sigma}(\mathbf{x}-\xi) d \xi .
\end{gathered}
$$

This theorem shows that the local force $F^{L}(\phi)$ has exactly opposite sign in $\omega$ (object) and in $\Omega \backslash \omega$ (background).

The following result together with Corollary 2 will be used in the proof of Theorem 7 mentioned later.

Corollary 4. Under the assumption of Theorem 3, one has the following. (i) If $P q-Q p \geq 0$, then

$$
\begin{gathered}
F^{L}(\phi) \geq S(P q-Q p) \frac{\left(g_{1}-g_{2}\right)^{2}}{P^{4} / 4}, \quad \text { in } \omega, \\
F^{L}(\phi) \leq-S(P q-Q p) \frac{\left(g_{1}-g_{2}\right)^{2}}{P^{4} / 4}, \quad \text { in } \Omega \backslash \omega .
\end{gathered}
$$

(ii) If $P q-Q p \leq 0$, then

$$
\begin{gathered}
F^{L}(\phi) \leq S(P q-Q p) \frac{\left(g_{1}-g_{2}\right)^{2}}{P^{4} / 4}, \quad \text { in } \omega, \\
F^{L}(\phi) \geq-S(P q-Q p) \frac{\left(g_{1}-g_{2}\right)^{2}}{P^{4} / 4}, \quad \text { in } \Omega \backslash \omega,
\end{gathered}
$$

where

$$
\left.S=\min \left\{K_{\sigma}(\mathbf{x}-\xi): \xi \in \Omega\right)\right\} .
$$

The proofs of Theorem 3 and Corollary 4 are similar to Theorem 1 and Corollary 2, respectively; see Appendix B for details.

3.3. Behavior of Our Model. In this section, we analyze the behavior of our model (10) for image segmentation. We will show that the zero-level line of the evolution function starting with a bounded function finally comes to a unique steady state, which separates the object from the background.

Due to the discrete nature of image, the continuous equation (10) can be discretized in space with space step 1 (implied pixel spacing), but also in time with a time step $\Delta t$ as follows:

$$
\begin{gathered}
\phi_{i, j}^{k+1}=\phi_{i, j}^{k}+\Delta t \cdot F\left(\phi_{i, j}^{k}\right), \\
0 \leq i \leq p-1,0 \leq j \leq q-1,
\end{gathered}
$$

where $p \times q$ corresponds to the image size, $I_{i, j}=I(i, j)$ and $\phi_{i, j}^{k}=\phi(k \Delta t, i, j)$ with $k \geq 0$ and $\phi_{i, j}^{0}=\phi_{0}(i, j)$.

Corollary 5. Let $M n_{0}-N_{0} m=|\Omega|\left|\omega \cap\left\{\phi^{0}>0\right\}\right|-\mid\left\{\phi^{0}>\right.$ $0\}|| \omega \mid \neq 0$.

(i) If $M n_{0}-N_{0} m>0$, then

$$
\begin{aligned}
F^{G}\left(\phi^{k}\right)>( & \left.M n_{0}-N_{0} m\right) \\
& \times \frac{\left(g_{1}-g_{2}\right)^{2}}{M^{4} / 4}=A>0, \quad \text { in } \omega
\end{aligned}
$$

$$
\left(k \geq 1, k \in z^{+}\right),
$$

$$
\begin{aligned}
F^{G}\left(\phi^{k}\right)<- & \left(M n_{0}-N_{0} m\right) \\
\times & \frac{\left(g_{1}-g_{2}\right)^{2}}{M^{4} / 4}=-A<0, \quad \text { in } \Omega \backslash \omega
\end{aligned}
$$

$$
\left(k \geq 1, k \in z^{+}\right) \text {. }
$$


(ii) If $M n_{0}-N_{0} m<0$, then

$$
\begin{aligned}
F^{G}\left(\phi^{k}\right)< & \left(M n_{0}-N_{0} m\right) \\
& \times \frac{\left(g_{1}-g_{2}\right)^{2}}{M^{4} / 4}=A<0, \text { in } \omega
\end{aligned}
$$$$
\left(k \geq 1, k \in z^{+}\right) \text {, }
$$

$$
\begin{aligned}
F^{G}\left(\phi^{k}\right)>- & \left(M n_{0}-N_{0} m\right) \\
\times \frac{\left(g_{1}-g_{2}\right)^{2}}{M^{4} / 4}=-A>0, \quad \text { in } \Omega \backslash \omega & \quad\left(k \geq 1, k \in z^{+}\right),
\end{aligned}
$$

where

$$
A=\left(M n_{0}-N_{0} m\right) \frac{\left(g_{1}-g_{2}\right)^{2}}{M^{4} / 8} .
$$

We provide the proof of Corollary 5 in Appendix C. Similar analysis can prove the following corollary, Corollary 6.

Corollary 6. Let $P q_{0}-Q_{0} p \neq 0$.

(i) If $P q_{0}-Q_{0} p>0$, then

$$
\begin{aligned}
F^{L}\left(\phi^{k}\right)> & \left(P q_{0}-Q_{0} p\right) \\
& \times \frac{\left(g_{1}-g_{2}\right)^{2}}{P^{4} / 4}=B>0, \quad \text { in } \omega \\
& \left(k \geq 1, k \in z^{+}\right), \\
F^{L}\left(\phi^{k}\right)<- & \left(P q_{0}-Q_{0} p\right) \\
& \times \frac{\left(g_{1}-g_{2}\right)^{2}}{P^{4} / 4}=-B<0, \quad \text { in } \Omega \backslash \omega \\
& \quad\left(k \geq 1, k \in z^{+}\right) .
\end{aligned}
$$

(ii) If $P q_{0}-Q_{0} p<0$, then

$$
\begin{aligned}
F^{L}\left(\phi^{k}\right)< & \left(P q_{0}-Q_{0} p\right) \\
& \times \frac{\left(g_{1}-g_{2}\right)^{2}}{P^{4} / 4}=B<0, \quad \text { in } \omega \\
F^{L}\left(\phi^{k}\right)>- & \left(P q_{0}-Q_{0} p\right) \\
& \times \frac{\left(g_{1}-g_{2}\right)^{2}}{P^{4} / 4}=-B>0, \quad \text { in } \Omega \backslash \omega \\
& \quad\left(k \geq 1, k \in z^{+}\right),
\end{aligned}
$$

where

$$
B=\left(P q_{0}-Q_{0} p\right) \frac{\left(g_{1}-g_{2}\right)^{2}}{P^{4} / 8} .
$$

Theorem 7. If $\phi^{0}(\mathbf{x})$ is a bounded function with $\left(M n_{0}-\right.$ $\left.N_{0} m\right)\left(P q_{0}-Q_{0} p\right)>0$, then there exists a positive integer $K$ such that, for $k \geq K$,

$\phi_{i, j}^{k+1}>0, \quad$ in $\omega$,

$\phi_{i, j}^{k+1}<0, \quad$ in $\Omega \backslash \omega, \quad$ if $M n_{0}-N_{0} m>0, P q_{0}-Q_{0} p>0$,

$\phi_{i, j}^{k+1}<0, \quad$ in $\omega$,

$\phi_{i, j}^{k+1}>0, \quad$ in $\Omega \backslash \omega$,

if $M n_{0}-N_{0} m<0, P q_{0}-Q_{0} p<0$.

We provide the proof of Theorem 7 in Appendix D.

The significance of Theorem 7 is that if we choose $\phi^{0}(\mathbf{x})$ such that $\left(M n_{0}-N_{0} m\right)\left(P q_{0}-Q_{0} p\right)>0$, then the zero-level line of $\phi(\mathbf{x})$ starting with such initial function $\phi^{0}(\mathbf{x})$ can finally come to a unique steady state, which separates object from the background.

Remark. Mathematically there does exist $\phi^{0}(\mathbf{x})$ such that $\left(M n_{0}-N_{0} m\right)\left(P q_{0}-Q_{0} p\right) \leq 0$; in practice, however, we always can guarantee that

$$
\left(M n_{0}-N_{0} m\right)\left(P q_{0}-Q_{0} p\right)>0 .
$$

3.4. Discussion of Initial Function. Theorem 7 guarantees that the proposed model computes a unique steady state regardless of the initialization; that is, the convergence of the zerolevel line $\{\phi=0\}$ is irrespective of the initial function. This means that we can obtain the same zero-level line in the steady state if we choose the initial function as a bounded function $\phi^{0}$ with $\left(M n_{0}-N_{0} m\right)\left(P q_{0}-Q_{0} p\right)>0$.

In applications, the initial function $\phi^{0}$ can be defined via a simple curve (closed curve or line segment) in image domain. For example, we can choose the initial function as a signed distance to a circle, which is widely used in most of image segmentation models with level set methods. For the proposed model, however, we prefer to define the initial function $\phi^{0}(\mathbf{x})$ as a piecewise constant or constant function as follows.

(1) If the curve $C$ is a closed curve (e.g., circle or square), then $\phi^{0}(\mathbf{x})$ is defined by

$$
\phi^{0}(\mathbf{x})= \begin{cases}+\rho, & (\mathbf{x}) \in \text { inside }(C), \\ -\rho, & (\mathbf{x}) \in \text { outside }(C),\end{cases}
$$

where $\rho \neq 0$ is a constant.

(2) If the curve $C$ is a line segment that partitions the image domain $\Omega$ into two disjoint regions $\Omega_{1}$ and $\Omega_{2}$ (e.g., the left and right half regions of image domain $\Omega$, respectively) with $\Omega=\Omega_{1} \cup \Omega_{2}$ and $\Omega_{1} \cap \Omega_{2}=\Phi$, then $\phi_{0}(x, y)$ is defined by

$$
\phi^{0}(\mathbf{x})= \begin{cases}+\rho, & (\mathbf{x}) \in \Omega_{1}, \\ -\rho, & (\mathbf{x}) \in \Omega_{2},\end{cases}
$$

where $\rho \neq 0$ is a constant. 
(3) We can also define a zero function as follows:

$$
\phi^{0}(\mathbf{x})=0 .
$$

Next, we prove the fact that, with $\phi^{0}(\mathbf{x})=0, \phi(\mathbf{x})$ becomes a sign-changing function and satisfy the condition of $\left(M n_{1}-\right.$ $\left.N_{1} m\right)\left(P q_{1}-Q_{1} p\right)>0$ after the first iteration.

Theorem 8. If the initial function $\phi^{0}(\mathbf{x})=0$ in $\Omega$ and

$$
\begin{gathered}
c_{1}^{0}(\mathbf{x})=f_{1}^{0}(\mathbf{x})=2 \cdot \operatorname{mean}_{\mathbf{x} \in \Omega} I(\mathbf{x}), \\
c_{2}^{0}(\mathbf{x})=f_{2}^{0}(\mathbf{x})=0,
\end{gathered}
$$

then after the first iteration, one has

$$
\operatorname{sign}(F(0))= \begin{cases}-1, & \mathbf{x} \in \Omega_{1}, \\ 1, & \mathbf{x} \in \Omega_{2},\end{cases}
$$

where $\Omega_{1} \cap \Omega_{2}=\Phi, \Omega_{1} \neq \Phi, \Omega_{2} \neq \Phi$.

We provide the proof of Theorem 8 in Appendix E. By (25) and Theorem 8 , we have

$$
\begin{aligned}
\phi^{1}(\mathbf{x})= & \delta_{\varepsilon}(0) \operatorname{mean}_{\mathbf{x} \in \Omega} I(\mathbf{x}) \\
& \times\left(I-\operatorname{mean}_{\mathbf{x} \in \Omega} I(\mathbf{x})\right) \begin{cases}<0, & \mathbf{x} \in \Omega_{1}, \\
>0, & \mathbf{x} \in \Omega_{2} .\end{cases}
\end{aligned}
$$

Therefore, $\phi^{1}(\mathbf{x})$ became a sign-changing function. Then, using two distinct gray levels of (13) and the above demonstration, we have

$$
\begin{aligned}
& \left(M n_{1}-N_{1} m\right)\left(P q_{1}-Q_{1} p\right) \\
& =\left(m\left(M-N_{1}\right)\right)\left(p\left(P-Q_{1}\right)\right) \\
& =m p\left(M-N_{1}\right)\left(P-Q_{1}\right)>0 .
\end{aligned}
$$

\section{Implementation and Experimental Results}

4.1. Implementation. In tradition PDE-based methods, a certain diffusion term is usually included in the evolution equation to regularize the evolving function, but which increases the computational cost. Recently, [16] proposed a novel scheme to regularize the evolving function, that is, Gaussian filtering the evolving function after each of iterations. We adopt this scheme to regularize the evolving function $u$ at each of iteration; that is, $\phi^{n}=G_{\rho} * \phi^{n}$, where $p$ controls the regularization strength. Such regularization procedure has some advantages over the traditional regularization term, such as computational efficiency and better smoothing effect; see [16] for more explanations.

The main procedures of the proposed algorithm can be summarized as follows.

(1) Initialize the evolving function $\phi^{0}(\mathbf{x})$.

(2) Compute $c_{i}(\phi)$ and $f_{i}(\phi),(i=1,2)$ using $(10)$.
(3) Evolve the function $\phi$ according to (11).

(4) Regularize the function $\phi$ with a Gaussian filter, that is; $\phi^{n}=G_{\rho} * \phi^{n}$.

(5) Check whether the evolution is finished. If not, return to step 2 .

4.2. Experimental Results. In this section, we show experimentally that the proposed model not only can provide desirable segmentation results in the presence of intensity inhomogeneity but also allows for more flexible initialization of the contour compared to the RSF and LIF models.

In our numerical experiments, for our model, we choose the parameters as follows: $\varepsilon=1.5$ for the regularized Dirac function, $\Delta t=0.025$ (time step), $h=1$ (space step), $\omega=0.1$, and $n=5$. The MATLAB source code of the LIF model algorithm is downloaded at http://www4.comp.polyu.edu .hk/ cslzhang/code/LIF.zip, and the RSF model algorithm is downloaded at http://www.engr.uconn.edu/ cmli/code/ RSF_v0_v0.1.rar. All experiments were run under MATLAB 2010b on a PC with Dual $2.7 \mathrm{GHz}$ processor.

We will utilize two region overlap metrics to evaluate the performances of the three models quantitatively. The two metrics are the ratio of segmentation error (RSE) [18] and the dice similarity coefficient (DSC) $[14,18,19]$. If $S_{1}$ and $S_{2}$ represent a given baseline foreground region (e.g., true object) and the foreground region found by the model, respectively, then the two metrics are defined as follows:

$$
\begin{gathered}
\mathrm{RSE}=\frac{N\left(S_{1} \backslash S_{2}\right)+N\left(S_{2} \backslash S_{1}\right)}{N(\Omega)}, \\
\mathrm{DSC}=\frac{2 N\left(S_{1} \cap S_{2}\right)}{N\left(S_{1}\right)+N\left(S_{2}\right)},
\end{gathered}
$$

where $N(\cdot)$ indicates the number of pixels in the enclosed region, and $\Omega$ is image domain. The closer the DSC value to 1 , the better the segmentation. Since $N\left(S_{1} \backslash S_{2}\right)+N\left(S_{2} \backslash S_{1}\right)$ is the number of the pixels mistakenly classified by the model, lower RSE means that there are fewer pixels misclassified; that is, the image can be segmented more accurately. Thus, a perfect segmentation will give DSC $=1, \mathrm{RSE}=0$.

In the first example (Figures 1-3), we mainly verify the computation of a unique steady state of the zero-level line of $\phi$, starting with three types of representative initial functions, that is, signed distance function, piecewise constant functions by (26)-(27), and zero function, respectively. The top row of Figure 1 shows the evolution of an active contour (i.e., zero-level line $\{\phi=0\}$ ), with the function $\phi$ initialized to a signed distance function, piecewise constant functions by (26)-(27) with $\rho=1$, and zero function, respectively, while the bottom row shows the corresponding evolution of $\phi$. With such initializations, the zero-level line of $\phi$ converges to the same steady state.

In Figure 2, we show that our model has the capability of detecting multiple objects or objects with interior holes or blurred edges, only starting with a zero function. The contours (zero-level lines) evolution processes are shown in the second column to the forth column. 

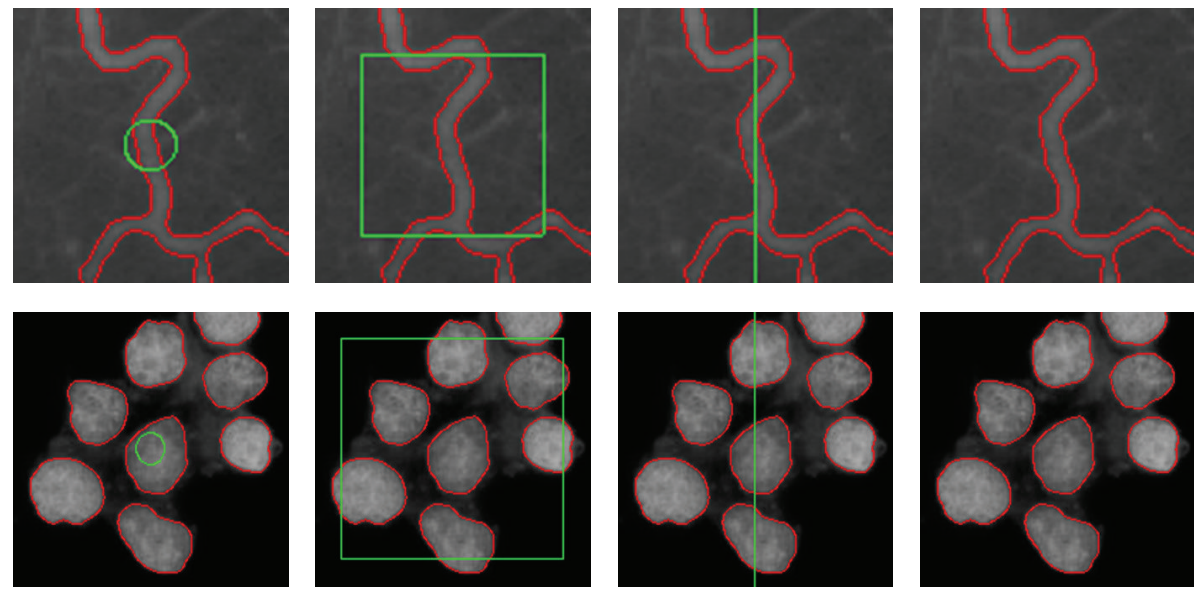

FIGURE 1: Segmentations of our model for two real images with $\phi$ initialized as different functions. Columns 1 to 4 : signed distance function, piecewise constant functions by (26)-(27) with $\rho=1$, and zero function.
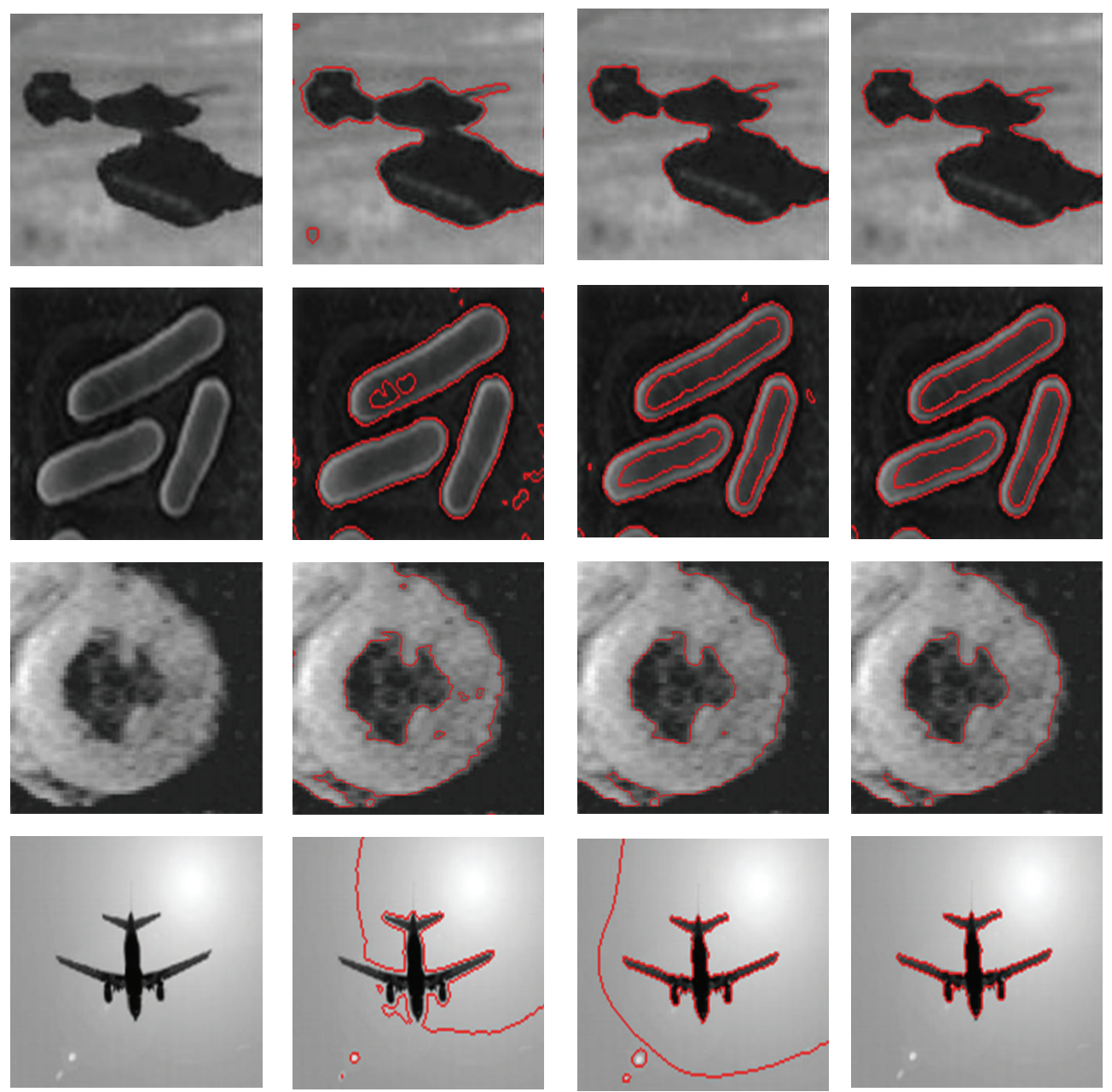

FIGURE 2: Applications of our model to four images (slug, cell, ventriculus sinister MR, and real plane images). The curve evolution process from the initial contour (in the first column) to the final contour (in the fourth column) is shown in every row for the corresponding image. 

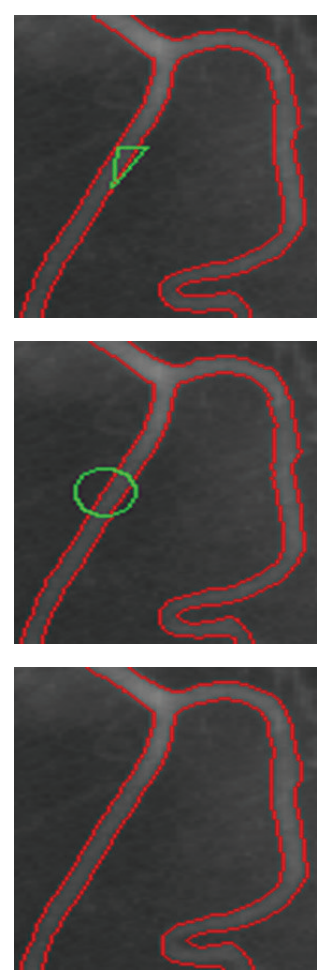

(a)
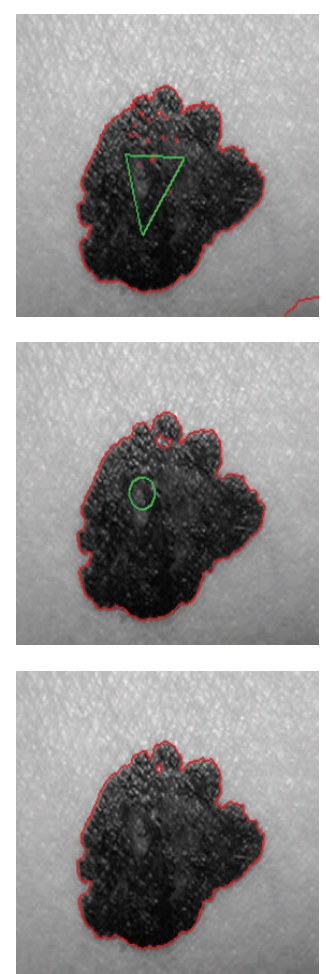

(b)
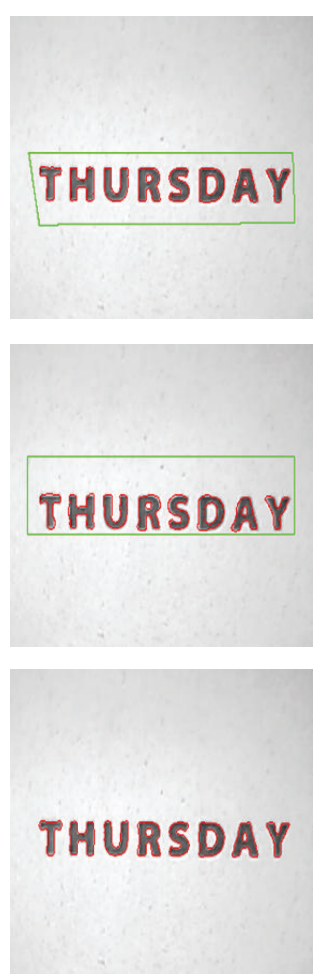

(c)
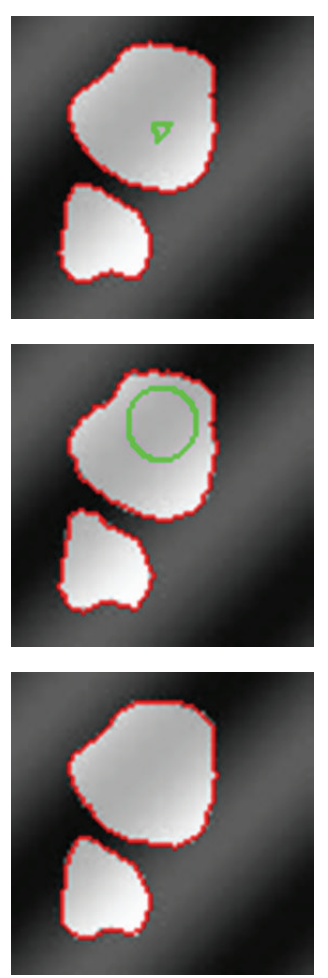

(d)

FIGURE 3: Comparisons of three models. Rows 1-3: RSF model, LIF model, and our model with $\phi_{0}=0$. For the RSF and LIF models, initial and final contours are shown in green and red color, respectively. Columns 1-4: the 4 pictures indicate (a), (b), (c), (d), respectively.
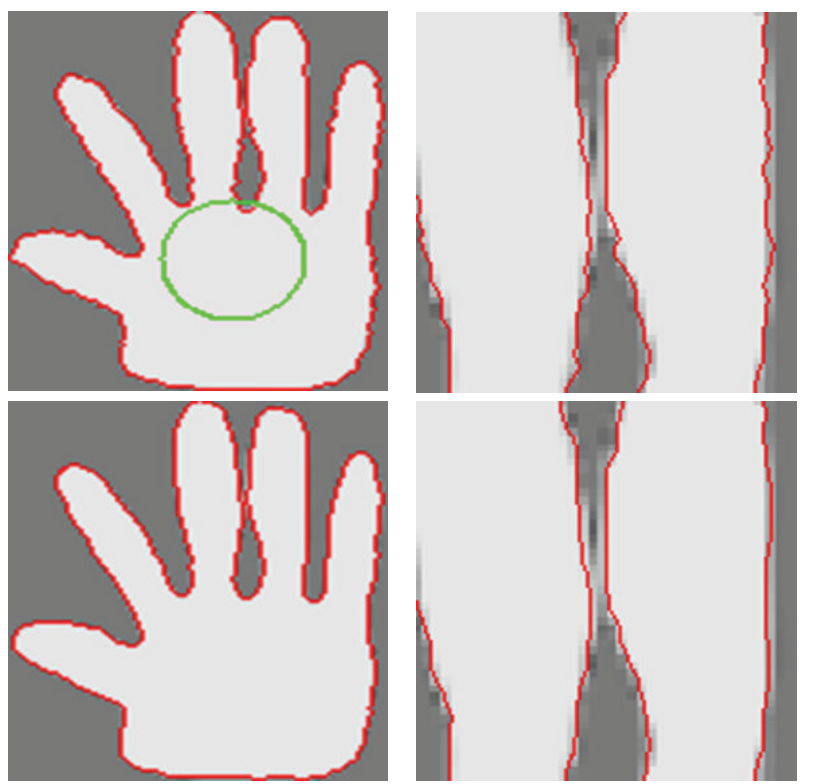

FIgURE 4: Segmentation results of both models for a hand phantom. Upper row: LIF model. Lower row: our model with $\phi_{0}=0$. Column 2: zoomed view of the narrow parts in hand fingers.

We choose the segmentation results of the RSF and LIF models as baseline foreground regions and then compute DSC values for the corresponding images. The RSE and DSC values for the four real images are given in Table 1 , from which
TABLE 1: RSE and DSC for RSF and LIF models and our model.

\begin{tabular}{lcccc}
\hline & \multicolumn{2}{c}{ RSF model } & \multicolumn{2}{c}{ LIF model } \\
& RSE (\%) & DSC (\%) & RSE (\%) & DSC (\%) \\
\hline Figure 3(a) & 0.27 & 97.17 & 2.99 & 98.91 \\
Figure 3(b) & 2.71 & 96.37 & 0.12 & 99.13 \\
Figure 3(c) & 0.49 & 87.95 & 0.45 & 88.06 \\
Figure 3(d) & 0.16 & 97.66 & 0.76 & 98.91 \\
\hline
\end{tabular}

we can see that the proposed algorithm works as well as the RSF and LIF models for images with intensity inhomogeneity.

The experimental results shown in Figure 4 validate that our method can also achieve subpixel segmentation accuracy as in [15]. As can be seen from Figures 4(b) and 4(d), both models achieve subpixel segmentation accuracy of the finger boundaries. The final contour accurately reflects the true hand shape.

\section{Conclusion}

In this study, we propose a new active contour model integrating a local intensity fitting (LIF) energy with an auxiliary global intensity fitting (GIF) energy. The LIF energy is responsible for attracting the contour toward object boundaries and is dominant near object boundaries, while the GIF energy incorporates global image information to improve the robustness to initialization of the contours. The proposed model can efficiently handle intensity inhomogeneity, while 
allowing for more flexible initialization and maintaining the subpixel accuracy. The utility model has the advantages of allowing for more flexible initialization of the contour and the capability of detecting multiple objects or objects with interior holes or blurred edges. But [14] has not been implemented.

\section{Appendices}

\section{A. Proofs of Theorem 1 and Corollary 2}

Proof of Theorem 1. Clearly, $\{\phi>0\} \neq \Phi$ and $\{\phi<0\} \neq \Phi$, owing to the sign-changing property of $\phi(x, y)$. Thus, by (3), we have

$$
\begin{aligned}
c_{1}(\phi) & =\frac{g_{1} n+g_{2}(N-n)}{N}=\frac{\left(g_{1}-g_{2}\right) n+g_{2} N}{N}, \\
c_{2}(\phi) & =\frac{g_{1}(m-n)+g_{2}[(M-N)-(m-n)]}{M-N} \\
& =\frac{\left(g_{1}-g_{2}\right)(m-n)+g_{2}(M-N)}{M-N},
\end{aligned}
$$

and so,

$$
\begin{aligned}
c_{1}(\phi)-c_{2}(\phi)= & \left((M-N)\left[\left(g_{1}-g_{2}\right) n+g_{2} N\right]\right. \\
& \left.-N\left[\left(g_{1}-g_{2}\right)(m-n)+g_{2}(M-N)\right]\right) \\
& \times(N(M-N))^{-1} \\
= & \frac{\left(g_{1}-g_{2}\right)(M n-N m)}{N(M-N)} .
\end{aligned}
$$

Therefore, in $\omega$, we get

$$
\begin{aligned}
r l F^{G}= & \left(c_{1}(\phi)-c_{2}(\phi)\right)\left(\left(g_{1}-c_{1}(\phi)\right) H_{\varepsilon}(\phi)\right. \\
& \left.+\left(g_{1}-c_{2}(\phi)\right)\left(1-H_{\varepsilon}(\phi)\right)\right) \\
= & \frac{\left(g_{1}-g_{2}\right)(M n-N m)}{N(M-N)} \cdot \frac{\left(g_{1}-g_{2}\right)}{N(M-N)} \\
& \times\left[(M-N)(N-n) H_{\varepsilon}\right. \\
= & \left.\quad+N((M+n)-(m+N))\left(1-H_{\varepsilon}\right)\right] \\
& \quad \times\left[(M-N)(N-n) H_{\varepsilon}\right. \\
& \left.\quad+N((M+n)-(m+N))\left(1-H_{\varepsilon}\right)\right],
\end{aligned}
$$

and in $\Omega \backslash \omega$, we have

$$
\begin{aligned}
& F^{G}(\phi)=\left(c_{1}(\phi)-c_{2}(\phi)\right)\left(\left(g_{2}-c_{1}(\phi)\right) H_{\varepsilon}(\phi)\right. \\
&\left.+\left(g_{2}-c_{2}(\phi)\right)\left(1-H_{\varepsilon}(\phi)\right)\right) \\
&= \frac{\left(g_{1}-g_{2}\right)(M n-N m)}{N(M-N)} \\
& \times\left[-\left(g_{1}-g_{2}\right)\right. \\
& \quad \times\left(n(M-N) H_{\varepsilon}(\phi)\right. \\
&\left.\quad+N(m-n)\left(1-H_{\varepsilon}(\phi)\right)\right) \\
&\left.\quad \times(N(M-N))^{-1}\right] \\
&=\frac{-(M n-N m)\left(g_{1}-g_{2}\right)^{2}}{N^{2}(M-N)^{2}} \\
& \quad \times\left[\left(n(M-N) H_{\varepsilon}(\phi) N(m-n)\left(1-H_{\mathcal{\varepsilon}}(\phi)\right)\right)\right] .
\end{aligned}
$$

This completes the proof of (14). The last assertion (15) follows clearly from the following fact:

$$
\begin{aligned}
(M-N)(N-n) H_{\varepsilon}(\phi) \\
\quad+N((M+n)-(m+N))\left(1-H_{\varepsilon}(\phi)\right) \\
\geq H_{\varepsilon}(\phi)+\left(1-H_{\varepsilon}(\phi)\right) \geq 1 \\
\left(n(M-N) H_{\varepsilon}(\phi)+N(m-n)\left(1-H_{\varepsilon}(\phi)\right)\right) \\
\geq H_{\varepsilon}(\phi)+\left(1-H_{\varepsilon}(\phi)\right) \geq 1 .
\end{aligned}
$$

The proof of Theorem 1 is completed.

Now, we give the proof of Corollary 2. (i) Since

$$
\begin{aligned}
M^{2} & =(N+(M-N))^{2} \\
& =N^{2}+(M-N)^{2}+2 N(M-N) \\
& \geq 4 N(M-N),
\end{aligned}
$$

by (14), (A.5), and (A.7) we have

$$
\begin{aligned}
F^{G}(\phi)=( & M n-N m)\left(g_{1}-g_{2}\right)^{2} \\
& \times\left[(M-N)(N-n) H_{\varepsilon}\right. \\
& \left.\quad+N((M+n)-(m+N))\left(1-H_{\varepsilon}\right)\right] \\
& \times\left(N^{2}(M-N)^{2}\right)^{-1} \\
\geq & (M n-N m) \frac{\left(g_{1}-g_{2}\right)^{2}}{M^{4} / 4}, \text { in } \omega,
\end{aligned}
$$


and by (14) and (A.6),

$$
\begin{aligned}
F^{G}(\phi)=- & (M n-N m)\left(g_{1}-g_{2}\right)^{2} \\
& \times\left[\left(n(M-N) H_{\varepsilon}\right.\right. \\
& \left.\left.+N(m-n)\left(1-H_{\varepsilon}\right)\right)\right] \\
& \times\left(N^{2}(M-N)^{2}\right)^{-1} \\
\leq & -(M n-N m) \frac{\left(g_{1}-g_{2}\right)^{2}}{M^{4} / 4}, \quad \text { in } \Omega \backslash \omega .
\end{aligned}
$$

This completes the proof of (17), and similarly for (18). The proof is completed.

\section{B. Proofs of Theorem 3 and Corollary 4}

Proof of Theorem 3. By (6), we have

$$
\begin{aligned}
& f_{1}(\mathbf{x})=\frac{\int_{\{\phi>0\}} K_{\sigma}(\mathbf{x}-\xi) I(\xi) d \xi}{\int_{\{\phi>0\}} K_{\sigma}(\mathbf{x}-\xi) d \xi} \\
& =\left(\int_{\{\phi>0\} \cap \omega} K_{\sigma}(\mathbf{x}-\xi) I(\xi) d \xi\right. \\
& \left.+\int_{\{\phi>0\} \backslash(\{\phi>0\} \cap \omega)} K_{\sigma}(\mathbf{x}-\xi) I(\xi) d \xi\right) \\
& \times\left(\int_{\{u>\bar{I}\}} K_{\sigma}(\mathbf{x}-\xi) d \xi\right)^{-1} \\
& =\left(g_{1} \int_{\{\phi>0\} \cap \omega} K_{\sigma}(\mathbf{x}-\xi) d \xi\right. \\
& \left.+g_{2} \int_{\{\phi>0\} \backslash(\{\phi>0\} \cap \omega)} K_{\sigma}(\mathbf{x}-\xi) d \xi\right) \\
& \times\left(\int_{\{u>\bar{I}\}} K_{\sigma}(x-\xi) d \xi\right)^{-1} \\
& =\frac{g_{1} q+g_{2}(Q-q)}{Q}, \\
& f_{2}(\mathbf{x})=\frac{\int_{\{\phi \leq 0\}} K_{\sigma}(\mathbf{x}-\xi) I(\xi) d \xi}{\int_{\{\phi \leq 0\}} K_{\sigma}(\mathbf{x}-\xi) d \xi} \\
& =\left(\int_{\{\phi \leq 0\} \cap \omega} K_{\sigma}(\mathbf{x}-\xi) I(\xi) d \xi\right. \\
& \left.+\int_{\{\phi \leq 0\} \backslash(\{\phi \leq 0\} \cap \omega)} K_{\sigma}(\mathbf{x}-\xi) I(\xi) d \xi\right)
\end{aligned}
$$

$$
\begin{aligned}
& \times\left(\int_{\{\phi \leq 0\}} K_{\sigma}(\mathbf{x}-\xi) d \xi\right)^{-1} \\
& =\left(g_{1} \int_{\{\phi \leq 0\} \cap \omega} K_{\sigma}(\mathbf{x}-\xi) d \xi\right. \\
& \left.\quad+g_{2} \int_{\{\phi \leq 0\} \backslash(\{\phi \leq 0\} \cap \omega)} K_{\sigma}(\mathbf{x}-\xi) d \xi\right) \\
& \times\left(\int_{\{\phi \leq 0\}} K_{\sigma}(\mathbf{x}-\xi) d \xi\right)^{-1} \\
& =\frac{g_{1}(p-q)+g_{2}[(P-Q)-(p-q)]}{P-Q},
\end{aligned}
$$

and so,

$$
f_{1}(\mathbf{x})-f_{2}(\mathbf{x})=\frac{\left(g_{1}-g_{2}\right)(P q-Q p)}{Q(P-Q)}
$$

Therefore, in $\omega$, we get

$$
\begin{aligned}
F^{L}(\phi)=( & \left.m_{1}(\phi)-m_{2}(\phi)\right) \\
& \times\left(\left(g_{1}-m_{1}(\phi)\right) H_{\varepsilon}(\phi)\right. \\
& \left.+\left(g_{1}-m_{2}(\phi)\right)\left(1-H_{\varepsilon}(\phi)\right)\right) \\
=( & P q-Q p)\left(g_{1}-g_{2}\right)^{2} \\
\times & {\left[(P-Q)(Q-q) H_{\varepsilon}(\phi)\right.} \\
& \left.+Q((P+q)-(p+Q))\left(1-H_{\varepsilon}(\phi)\right)\right] \\
\times & \left(Q^{2}(P-Q)^{2}\right)^{-1},
\end{aligned}
$$

and in $\Omega \backslash \omega$, we have

$$
\begin{aligned}
F^{L}= & \left(m_{1}(\phi)-m_{2}(\phi)\right) \\
& \times\left(\left(g_{2}-m_{1}(\phi)\right) H_{\varepsilon}(\phi)\right. \\
& \left.\quad+\left(g_{2}-m_{2}(\phi)\right)\left(1-H_{\varepsilon}(\phi)\right)\right) \\
= & -(P q-Q p)\left(g_{1}-g_{2}\right)^{2} \\
& \times\left[q(P-Q) H_{\varepsilon}(\phi)+Q(p-q)\left(1-H_{\varepsilon}(\phi)\right)\right] \\
& \times\left(Q^{2}(P-Q)^{2}\right)^{-1} .
\end{aligned}
$$


This completes the proof of (19). The last assertion (20) follows clearly from the following fact:

$$
\begin{aligned}
& (P-Q)(Q-q) H_{\varepsilon}(\phi) \\
& +Q((P+q)-(p+Q))\left(1-H_{\varepsilon}(\phi)\right) \\
& =H_{\varepsilon}(\phi)\left(\int_{\Omega}-\int_{\{\phi>0\}}\right) K_{\sigma}(\mathbf{x}-\xi) d \xi \\
& \cdot\left(\int_{\{\phi>0\}}-\int_{\{\phi>0\} \cap \omega}\right) K_{\sigma}(\mathbf{x}-\xi) d \xi \\
& +\left(1-H_{\varepsilon}(\phi)\right) \int_{\{\phi>0\}} K_{\sigma}(\mathbf{x}-\xi) d \xi \\
& \cdot\left(\left(\int_{\Omega}+\int_{\{\phi>0\} \cap \omega}\right)-\left(\int_{\omega}+\int_{\{\phi>0\}}\right)\right) K_{\sigma}(\mathbf{x}-\xi) d \xi \\
& =H_{\varepsilon}(\phi) \int_{\{\phi \leq 0\}} K_{\sigma}(\mathbf{x}-\xi) d \xi \\
& \cdot\left(\int_{\{\phi>0\} \backslash(\{\phi>0\} \cap \omega)} K_{\sigma}(\mathbf{x}-\xi) d \xi\right) \\
& +\left(1-H_{\varepsilon}(\phi)\right) \\
& \times \int_{\{\phi>0\}} K_{\sigma}(\mathbf{x}-\xi) d \xi \cdot\left(\int_{\{\phi \leq 0\} \backslash(\{\phi \leq 0\} \cap \omega)} K_{\sigma}(\mathbf{x}-\xi) d \xi\right) \\
& \geq H_{\varepsilon}(\phi)\left(\int_{\{\phi \leq 0\}} S d \xi\right) \cdot\left(\int_{\{\phi>0\} \backslash(\{\phi>0\} \cap \omega)} S d \xi\right) \\
& +\left(1-H_{\varepsilon}(\phi)\right)\left(\int_{\{\phi>0\}} S d \xi\right) \cdot\left(\int_{\{\phi \leq 0\} \backslash(\{\phi \leq 0\} \cap \omega)} S d \xi\right) \\
& =S\left[(M-N)(N-n) H_{\varepsilon}(\phi)\right. \\
& \left.+N((M-N)-(m-n))\left(1-H_{\varepsilon}(\phi)\right)\right] \\
& \geq S \text {, }
\end{aligned}
$$

$$
\begin{aligned}
q( & -Q) H_{\varepsilon}(\phi)+Q(p-q)\left(1-H_{\mathcal{\varepsilon}}(\phi)\right) \\
= & H_{\varepsilon}(\phi) \int_{\{\phi>0\} \cap \omega} K_{\sigma}(\mathbf{x}-\xi) d \xi \cdot \int_{\{\phi \leq 0\}} K_{\sigma}(\mathbf{x}-\xi) d \xi \\
& +\left(1-H_{\varepsilon}(\phi)\right) \\
& \times \int_{\{\phi>0\}} K_{\sigma}(\mathbf{x}-\xi) d \xi \cdot \int_{\{\phi \leq 0\} \cap \omega} K_{\sigma}(\mathbf{x}-\xi) d \xi \\
\geq & H_{\mathcal{\varepsilon}}(\phi)\left(\int_{\{u>\bar{I}\} \cap \omega} S d \xi\right) \cdot\left(\int_{\{u \leq \bar{I}\}} S d \xi\right) \\
& +\left(1-H_{\varepsilon}(\phi)\right)\left(\int_{\{u>\bar{I}\}} S d \xi\right) \cdot\left(\int_{\{u \leq \bar{I}\} \cap \omega} S d \xi\right) \\
= & S\left[n(M-N) H_{\varepsilon}(\phi)+\left(1-H_{\varepsilon}(\phi)\right) N(m-n)\right] \\
\geq & S
\end{aligned}
$$

where $\left.S=\min \left\{K_{\sigma}(\mathbf{x}-\xi): \xi \in \Omega\right)\right\}$.
Now, we give the proof of Corollary 4. (i) Since

$$
P^{2} \geq 4 P(P-Q)
$$

we have by (19) and (B.6)

$$
\begin{aligned}
F^{L}(\phi)= & (P q-Q p)\left(g_{1}-g_{2}\right)^{2} \\
& \times\left[(P-Q)(Q-q) H_{\varepsilon}(\phi)\right. \\
& \left.\quad+Q((P+q)-(p+Q))\left(1-H_{\varepsilon}(\phi)\right)\right] \\
& \times\left(Q^{2}(P-Q)^{2}\right)^{-1} \\
\geq & (P q-Q p) \frac{\left(g_{1}-g_{2}\right)^{2}}{P^{4} / 4}, \quad \text { in } \omega,
\end{aligned}
$$

and by (19) and (B.7),

$$
\begin{aligned}
F^{L}(\phi)= & -(P q-Q p)\left(g_{1}-g_{2}\right)^{2} \\
& \times\left[q(P-Q) H_{\varepsilon}(\phi)\right. \\
& \left.+Q(p-q)\left(1-H_{\varepsilon}(\phi)\right)\right] \\
& \times\left(Q^{2}(P-Q)^{2}\right)^{-1} \\
\leq & -(P q-Q p) \frac{\left(g_{1}-g_{2}\right)^{2}}{P^{4} / 4}, \quad \text { in } \Omega \backslash \omega .
\end{aligned}
$$

This completes the proof of (19), and similarly to (20). The proof is completed.

\section{Proof of Corollary 5}

Proof. (i) By Corollary 2 (i), we have

$$
\begin{array}{r}
F^{G}\left(\phi^{0}\right) \geq\left(M n_{0}-N_{0} m\right) \frac{\left(g_{1}-g_{2}\right)^{2}}{M^{4} / 4}=A>0, \\
\quad \text { in } \omega, \\
F^{G}\left(\phi^{0}\right) \leq-\left(M n_{0}-N_{0} m\right) \frac{\left(g_{1}-g_{2}\right)^{2}}{M^{4} / 4}=-A<0, \\
\text { in } \Omega \backslash \omega .
\end{array}
$$

At the first iteration, by (25) and (C.1), we have

$$
\begin{aligned}
\phi_{i, j}^{1}= & \phi_{i, j}^{0}+\Delta t \\
& \cdot F^{G}\left(\phi_{i, j}^{0}\right) \begin{cases}\geq \phi_{i, j}^{0}+\Delta t \cdot \delta_{\varepsilon}\left(\phi^{0}\right) A, & \text { in } \omega, \\
\leq \phi_{i, j}^{0}-\Delta t \cdot \delta_{\varepsilon}\left(\phi^{0}\right) A, & \text { in } \Omega \backslash \omega,\end{cases}
\end{aligned}
$$


or equivalently,

$$
\begin{gathered}
\phi^{1}(\mathbf{x}) \geq \phi^{0}(\mathbf{x})+\Delta t \cdot \delta_{\varepsilon}\left(\phi^{0}\right) A>\phi^{0}(\mathbf{x}), \quad \text { in } \omega, \\
\phi^{1}(\mathbf{x}) \leq \phi^{0}(\mathbf{x})-\Delta t \cdot \delta_{\varepsilon}\left(\phi^{0}\right) A<\phi^{0}(\mathbf{x}), \quad \text { in } \Omega \backslash \omega,
\end{gathered}
$$

which implies that

$$
\begin{aligned}
& M n_{1}-N_{1} m=|\Omega|\left|\omega \cap\left\{\phi^{1}>0\right\}\right|-\left|\left\{\phi^{1}>0\right\}\right||\omega| \\
& =(|\Omega \backslash \omega|+|\omega|)\left|\omega \cap\left\{\phi^{1}>0\right\}\right| \\
& -\left(\left|\omega \cap\left\{\phi^{1}>0\right\}\right|\right. \\
& \left.+\left|(\Omega \backslash \omega) \cap\left\{\phi^{1}>0\right\}\right|\right)|\omega| \\
& =|\Omega \backslash \omega|\left|\omega \cap\left\{\phi^{1}>0\right\}\right| \\
& -\left|(\Omega \backslash \omega) \cap\left\{\phi^{1}>0\right\}\right||\omega| \\
& >|\Omega \backslash \omega|\left|\omega \cap\left\{\phi^{0}>0\right\}\right| \\
& -\left|(\Omega \backslash \omega) \cap\left\{\phi^{0}>0\right\}\right||\omega| \\
& =|\Omega \backslash \omega|\left|\omega \cap\left\{\phi^{0}>0\right\}\right| \\
& +\left|\omega \cap\left\{\phi^{0}>0\right\}\right||\omega| \\
& -\left|\omega \cap\left\{\phi^{0}>0\right\}\right||\omega| \\
& -\left|(\Omega \backslash \omega) \cap\left\{\phi^{0}>0\right\}\right||\omega| \\
& =(|\Omega \backslash \omega|+|\omega|)\left|\omega \cap\left\{\phi^{0}>0\right\}\right| \\
& -\left(\left|\omega \cap\left\{\phi^{0}>0\right\}\right|\right. \\
& \left.+\left|(\Omega \backslash \omega) \cap\left\{\phi^{0}>0\right\}\right|\right)|\omega| \\
& =|\Omega|\left|\omega \cap\left\{\phi_{0}>0\right\}\right|-\left|\left\{\phi_{0}>0\right\}\right||\omega| \\
& =M n_{0}-N_{0} m>0 \text {. }
\end{aligned}
$$

In a manner similar to the steps used to obtain (26)-(27) and (C.1)- (C.4), we obtain

$$
\begin{gathered}
F^{G}\left(I, \phi^{1}\right) \geq\left(M n_{1}-N_{1} m\right) \frac{\left(g_{1}-g_{2}\right)^{2}}{M^{4} / 4} \\
>\left(M n_{0}-N_{0} m\right) \frac{\left(g_{1}-g_{2}\right)^{2}}{M^{4} / 4}=A>0, \quad \text { in } \omega, \\
F^{G}\left(I, \phi^{1}\right) \leq-\left(M n_{1}-N_{1} m\right) \frac{\left(g_{1}-g_{2}\right)^{2}}{M^{4} / 4} \\
<-\left(M n_{0}-N_{0} m\right) \frac{\left(g_{1}-g_{2}\right)^{2}}{M^{4} / 4}=-A<0, \\
\phi^{2}(\mathbf{x}) \geq \phi^{1}(\mathbf{x})+\Delta t \cdot \delta_{\varepsilon}\left(\phi^{1}\right) A>\phi^{1}(\mathbf{x}), \quad \text { in } \quad \omega \backslash \omega, \\
\phi^{2}(\mathbf{x}) \leq \phi^{1}(\mathbf{x})-\Delta t \cdot \delta_{\varepsilon}\left(\phi^{1}\right) A<\phi^{1}(\mathbf{x}), \quad \text { in } \quad \Omega \backslash \omega, \\
M n_{2}-N_{2} m>M n_{1}-N_{1} m>M n_{0}-N_{0} m>0 .
\end{gathered}
$$

\section{Proof of Theorem 7}

Proof. We prove (32), and similarly to (33). By (25), Corollary 5(i), and Corollary 6(i), we get

$$
\begin{aligned}
& \phi_{i, j}^{k+1}= \phi_{i, j}^{k}+\Delta t \cdot F\left(\phi_{i, j}^{k}\right) \\
&= \phi_{i, j}^{k}+\Delta t \\
& \cdot\left((1-\omega) F^{L}\left(\phi_{i, j}^{k}\right)+\omega F^{G}\left(\phi_{i, j}^{k}\right)\right) \\
& \geq \phi_{i, j}^{k}+\Delta t \\
& \cdot\left((1-\omega) B \cdot \delta_{\varepsilon}\left(\phi^{k+1}\right)+\omega A \cdot \delta_{\varepsilon}\left(\phi^{k+1}\right)\right) \\
&= \phi_{i, j}^{k}+((1-\omega) B+\omega A) \\
& \cdot \delta_{\varepsilon}\left(\phi^{k+1}\right) \cdot \Delta t, \quad \text { in } \omega \\
& \quad\left(k \geq 1, \quad k \in z^{+}\right), \\
& \leq \phi_{i, j}^{k}+\Delta t \\
& \cdot\left(-\left((1-\omega) B \cdot \delta_{\varepsilon}\left(\phi^{k+1}\right)\right.\right. \\
&\left.\left.+\omega A \cdot \delta_{\varepsilon}\left(\phi^{k+1}\right)\right)\right) \\
&= \phi_{i, j}^{k}-((1-\omega) B+\omega A) \\
& \delta_{\varepsilon}\left(\phi^{k+1}\right) \cdot \Delta t, \quad \text { in } \Omega \backslash \omega \\
& \quad\left(k \geq 1, \quad k \in z^{+}\right), \\
&
\end{aligned}
$$

which implies that

$$
\begin{array}{r}
\phi_{i, j}^{k+1} \geq \phi_{i}^{0}+((1-\omega) B+\omega A \cdot) \cdot \prod_{i=0}^{k+1} \delta_{\varepsilon}\left(\phi^{i}\right) \cdot \Delta t, \\
\text { in } \omega, \\
\phi_{i, j}^{k+1} \leq \phi_{i}^{0}-(k+1) A((1-\omega) B+\omega A \cdot) \cdot \prod_{i=0}^{k+1} \delta_{\varepsilon}\left(\phi^{i}\right) \cdot \Delta t, \\
\text { in } \Omega \backslash \omega .
\end{array}
$$

Because $\phi_{0}(\mathbf{x})$ is bounded in domain $\Omega$, there clearly exists a positive integer $K$ such that

$$
\begin{array}{ll}
\phi_{i, j}^{k+1}>0, & \text { in } \omega, \\
\phi_{i, j}^{k+1}<0, & \text { in } \Omega \backslash \omega,
\end{array} \quad \text { for } k \geq K .
$$

The proof is completed. 


\section{E. Proof of Theorem 8}

Proof. From (11), we have

$$
\begin{aligned}
F(0)= & \delta_{\varepsilon}(0)\left[\omega\left(\left(I-I^{G}\right) \cdot 2 \operatorname{mean}_{\mathbf{x} \in \Omega} I(\mathbf{x})\right)\right. \\
& \left.+(1-\omega)\left(\left(I-I^{L}\right) \cdot 2 \operatorname{mean}_{\mathbf{x} \in \Omega} I(\mathbf{x})\right)\right] \\
= & 2 \delta_{\varepsilon}(0) \operatorname{mean}_{\mathbf{x} \in \Omega} I(\mathbf{x})\left(I-2 H_{\varepsilon}(0) \operatorname{mean}_{\mathbf{x} \in \Omega} I(\mathbf{x})\right) \\
= & \delta_{\varepsilon}(0) \operatorname{mean}_{\mathbf{x} \in \Omega} I(\mathbf{x})\left(I-\operatorname{mean}_{\mathbf{x} \in \Omega} I(\mathbf{x})\right) .
\end{aligned}
$$

Clearly,

$$
\min _{\mathbf{x} \in \Omega} I(\mathbf{x})<\operatorname{mean}_{\mathbf{x} \in \Omega} I(\mathbf{x})<\max _{\mathbf{x} \in \Omega} I(\mathbf{x})
$$

which implies that

$$
\operatorname{sign}(F(0))= \begin{cases}-1, & \mathbf{x} \in \Omega_{1}, \\ 1, & \mathbf{x} \in \Omega_{2},\end{cases}
$$

where

$$
\begin{aligned}
& \Omega_{1}=\left\{I(\mathbf{x})<\operatorname{mean}_{\mathbf{x} \in \Omega} I(\mathbf{x})\right\}, \\
& \Omega_{2}=\left\{I(\mathbf{x})>\operatorname{mean}_{\mathbf{x} \in \Omega} I(\mathbf{x})\right\} .
\end{aligned}
$$

The proof of Theorem 8 is completed.

\section{Acknowledgments}

The authors would like to thank the anonymous reviewers for their valuable comments and suggestions to improve this paper. Besides, this work was supported by the Fundamental Research Funds for the Central University, Grant no. (CDJXS10100006).

\section{References}

[1] V. Caselles, F. Catté, T. Coll, and F. Dibos, "A geometric model for active contours in image processing," Numerische Mathematik, vol. 66, no. 1, pp. 1-31, 1993.

[2] T. Chan, M. Moelich, and B. Sandberg, "Some recent developments in variational image segmentation," UCLA CAM Report cam 06-52, 2006.

[3] L. He, Z. Peng, B. Everding et al., "A comparative study of deformable contour methods on medical image segmentation," Image and Vision Computing, vol. 26, no. 2, pp. 141-163, 2008.

[4] C. Li, C. Xu, C. Gui, and M. D. Fox, "Level set evolution without re-initialization: a new variational formulation," in Proceedings of the IEEE Computer Society Conference on Computer Vision and Pattern Recognition (CVPR '05), pp. 430-436, San Diego, Calif, USA, June 2005.

[5] C. Li, C. Xu, C. Gui, and M. D. Fox, "Distance regularized level set evolution and its application to image segmentation," IEEE Transactions on Image Processing, vol. 19, no. 12, pp. 3243-3254, 2010.
[6] B. Zhou and C. L. Mu, "Level set evolution for boundary extraction based on a p-Laplace equation," Applied Mathematical Modelling, vol. 34, no. 12, pp. 3910-3916, 2010.

[7] Y. Wang and C. He, "Adaptive level set evolution starting with a constant function," Applied Mathematical Modelling, vol. 36, no. 7, pp. 3217-3228, 2012.

[8] T. F. Chan and L. A. Vese, "Active contours without edges," IEEE Transactions on Image Processing, vol. 10, no. 2, pp. 266-277, 2001.

[9] A. Tsai, A. Yezzi, and A. S. Willsky, "Curve evolution implementation of the Mumford-Shah functional for image segmentation, denoising, interpolation, and magnification," IEEE Transactions on Image Processing, vol. 10, no. 8, pp. 1169-1186, 2001.

[10] T. Brox and D. Cremers, "On the statistical interpretation of the piecewise smooth Mumford-Shah functional," in Proceedings of the International Conference on Scale Space and Variational Methods in Computer Vision (SSVM '07), pp. 203-213, Ischia, Italy, 2007.

[11] C. Li, C. Y. Kao, J. C. Gore, and Z. Ding, "Implicit active contours driven by local binary fitting energy," in Proceedings of the IEEE Computer Society Conference on Computer Vision and Pattern Recognition (CVPR '07), pp. 1-7, Washington, DC, USA, June 2007.

[12] C. Li, C. Y. Kao, J. C. Gore, and Z. Ding, "Minimization of region-scalable fitting energy for image segmentation," IEEE Transactions on Image Processing, vol. 17, no. 10, pp. 1940-1949, 2008.

[13] S. Lankton and A. Tannenbaum, "Localizing region-based active contours," IEEE Transactions on Image Processing, vol. 17, no. 11, pp. 2029-2039, 2008.

[14] L. Wang, C. Li, Q. Sun, D. Xia, and C. Y. Kao, "Active contours driven by local and global intensity fitting energy with application to brain MR image segmentation," Computerized Medical Imaging and Graphics, vol. 33, no. 7, pp. 520-531, 2009.

[15] K. Zhang, H. Song, and L. Zhang, "Active contours driven by local image fitting energy," Pattern Recognition, vol. 43, no. 4, pp. 1199-1206, 2010.

[16] K. Zhang, L. Zhang, H. Song, and W. Zhou, "Active contours with selective local or global segmentation: a new formulation and level set method," Image and Vision Computing, vol. 28, no. 4, pp. 668-676, 2010.

[17] Y. Yuan and C. He, "Variational level set methods for image segmentation based on both $L^{2}$ and Sobolev gradients," Nonlinear Analysis. Real World Applications, vol. 13, no. 2, pp. 959-966, 2012.

[18] B. Liu, H. D. Cheng, J. Huang, J. Tian, X. Tang, and J. Liu, "Probability density difference-based active contour for ultrasound image segmentation," Pattern Recognition, vol. 43, no. 6 , pp. 2028-2042, 2010.

[19] D. W. Shattuck, S. R. Sandor-Leahy, K. A. Schaper, D. A. Rottenberg, and R. M. Leahy, "Magnetic resonance image tissue classification using a partial volume model," NeuroImage, vol. 13, no. 5, pp. 856-876, 2001. 


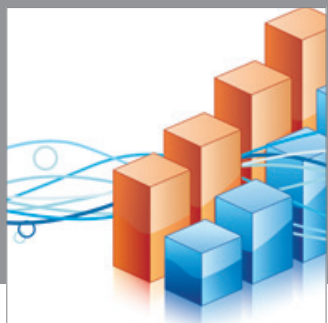

Advances in

Operations Research

mansans

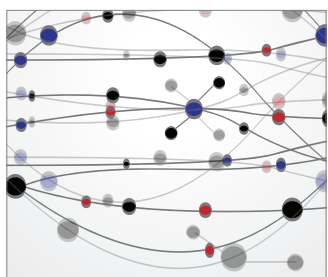

The Scientific World Journal
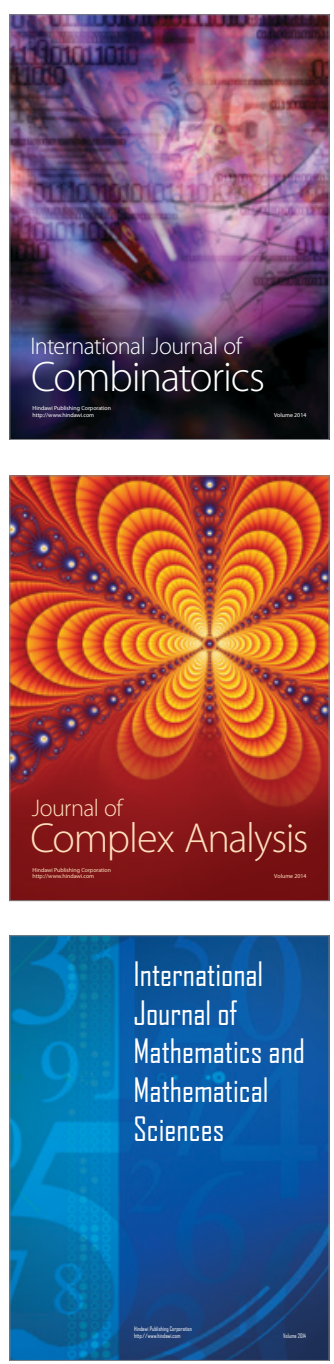
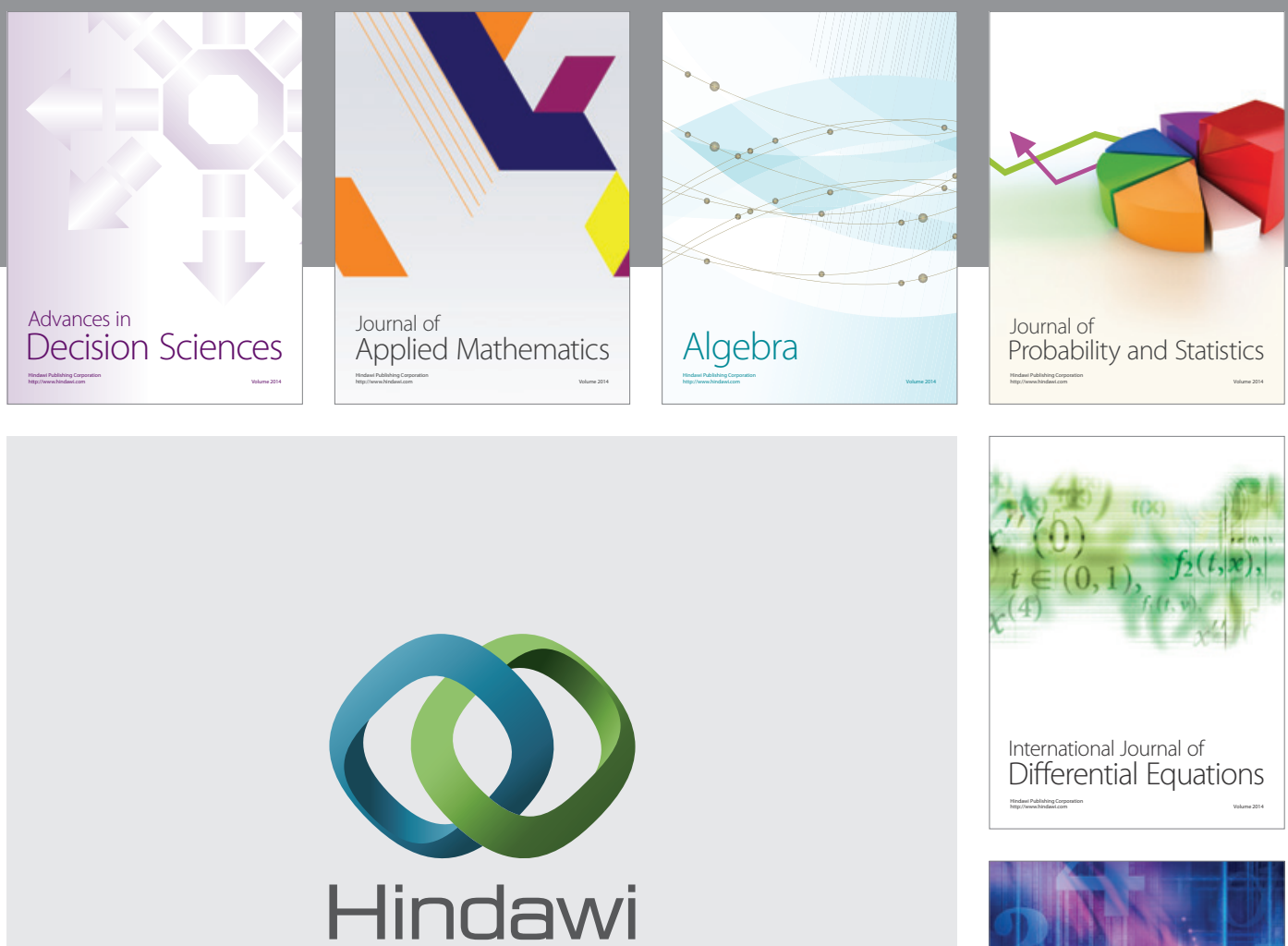

Submit your manuscripts at http://www.hindawi.com
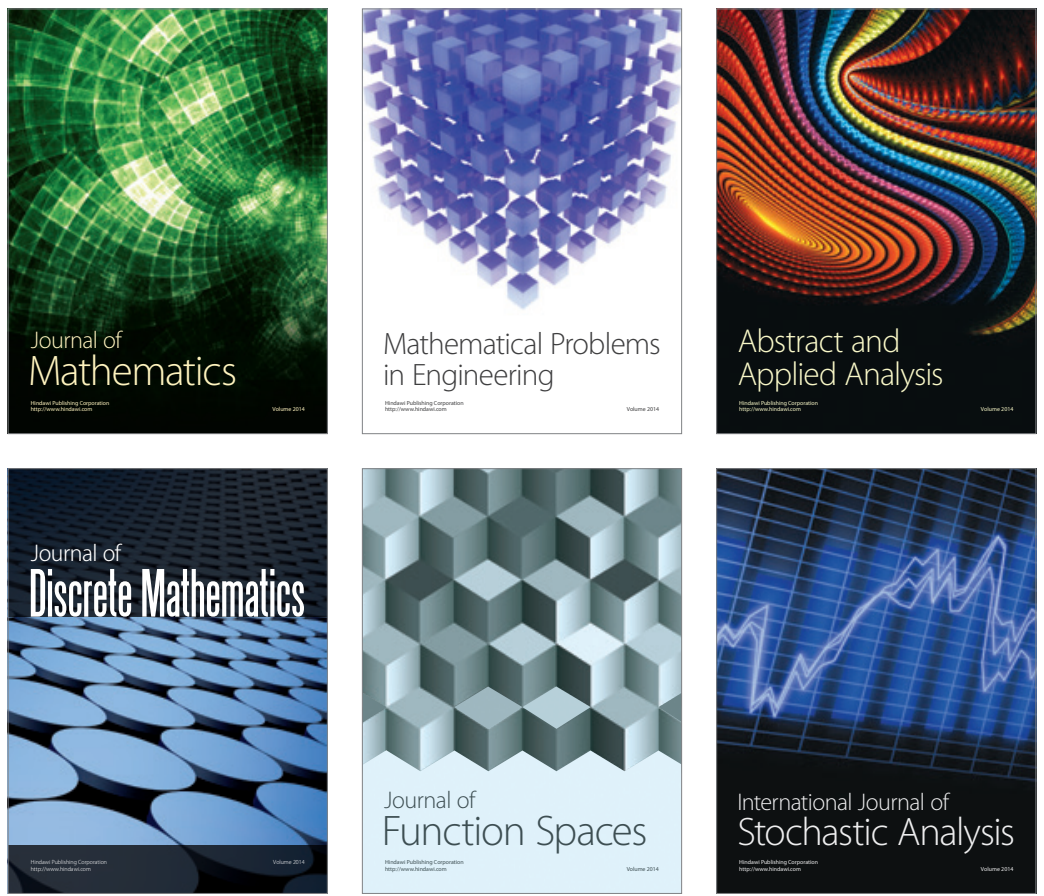

Journal of

Function Spaces

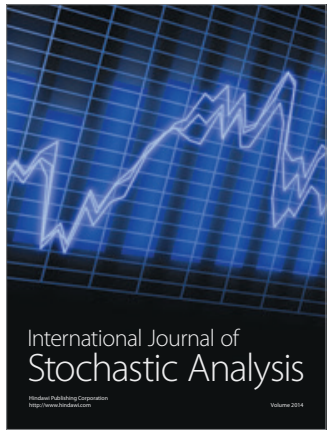

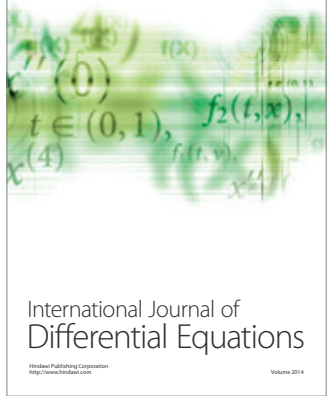
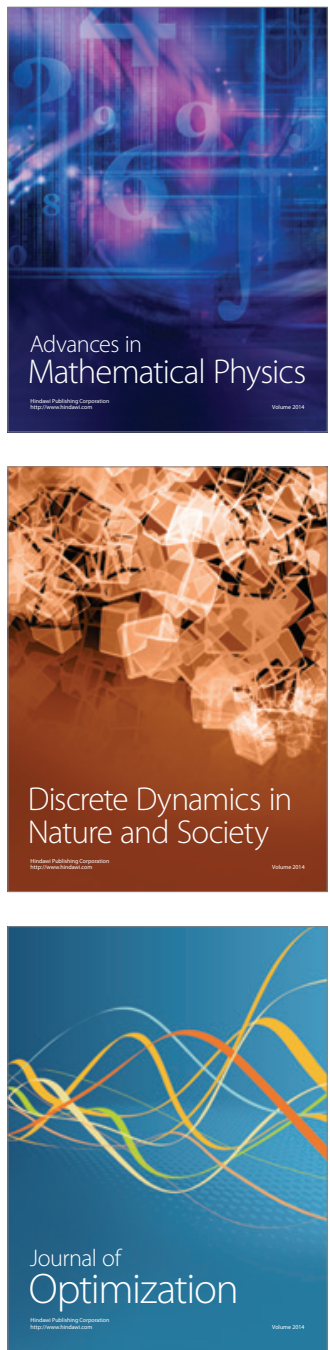